\title{
Relativistic prediction of the ground state of atomic Lawrencium
}

\author{
J.-P. Desclaux and B. Fricke $(*)$ \\ Centre d'Etudes Nucléaires de Grenoble, DRF-LIH, 85X, 38041 Grenoble Cedex, France \\ (Reçu le 4 février 1980, accepté le 28 avril 1980)
}

\begin{abstract}
Résumé. - Contrairement à la systéma tique du tableau périodique, mais en accord avec les suggestions antérieures de Brewer et de Mann, la configuration de l'état fondamental de l'atome de Lawrencium $(Z=103)$ n'est pas $7 \mathrm{~s}^{2} 6 \mathrm{~d}^{2} \mathrm{D}_{3 ; 2}$ mais $7 \mathrm{~s}^{2} 7 \mathrm{p}^{2} \mathrm{P}_{1 / 2}$. Cet écart à l'évolution normale à travers le tableau périodique est dû à l'importance des effets relativistes sur la couche la plus externe $7 \mathrm{p}_{1 / 2}$. On donne les résultats obtenus par la méthode multiconfigurationnelle Dirac-Fock pour l'atome de Lawrencium et certains atomes similaires plus légers. Ces calculs incluent les contributions magnétiques et de retard ainsi qu'une estimation des corrections dues à l'électrodynamique quantique.
\end{abstract}

\begin{abstract}
In contradiction to the prediction of the Periodic Table but in agreement with earlier suggestions by Brewer and Mann, the ground state configuration of atomic Lawrencium $(Z=103)$ will not be $7 \mathrm{~s}^{2} 6 \mathrm{~d}^{2} \mathrm{D}_{3 / 2}$ but $7 \mathrm{~s}^{2} 7 \mathrm{p}^{2} \mathrm{P}_{1 / 2}$. The reason for this deviation from normal trends across the Periodic Table are strong relativistic effects on the outermost $7 \mathrm{p}_{1 / 2}$ orbital. Multiconfiguration Dirac-Fock calculations are reported for Lawrencium and analogous lighter atoms. These calculations include contributions from magnetic and retardation interactions and an estimation of quantum electrodynamic corrections.
\end{abstract}

Introduction. - It is well-known that relativistic effects play an important role in the interpretation of inner shell phenomena [1]. During the last years, one also became more and more aware of the relativistic influences on outer shell problems like atomic radii [2], oscillator strength [3], photo-electron spectroscopy [4] etc. From the interpretation of relativistic SCF calculations of heavy atoms [5] it became obvious that chemistry is also partly affected by relativistic effects, even in the known region of elements like the main valency two of lead.

Up to now, no element is known whose ground state is different compared to the normal continuation of the Periodic Table [6], due to direct relativistic influences. One possible candidate for such a deviation is element Lawrencium $(Z=103)$. Every Periodic Table shows $5 \mathrm{f}^{14} 7 \mathrm{~s}^{2} 6 \mathrm{~d}^{1}$ as the ground state configuration. It was Mann [7] who first pointed out that single configuration Dirac-Fock calculations give $5 f^{14} 7 s^{2} 7 p^{1}$ as the ground state. Independently Brewer [8] came to the same conclusion using a semi-empirical extrapolation. Subsequent detailed investigations came to different conclusions [9-12], because the

$\left(^{*}\right)$ Permanent address : Physics Department, University of Kassel, D-3500 Kassel, West Germany. difference between the two possible ground states was found to be very small. A good theoretical prediction is very important, not only as a guide to the complicated experiment, but also as an excellent check for relativistic influences on outer shells and of the quality of the calculations.

Because the splitting between the two configurations is expected to be small, single configuration DiracFock calculations may give the wrong ordering of the levels if correlation effects, neglected in such calculations, have rather different contributions to the energy of the two states under consideration. To take the influence of these correlation effects partially into account, we have used the multiconfiguration DiracFock (MCDF) method [13], which we briefly summarize in the first section.

1. Method of calculation. - It is well known that the multiconfiguration method [14] is an efficient one to take into account most of the correlation effects while retaining only a reasonable number of configurations. In its relativistic version it has the further advantage of working $a b$ initio in the intermediate coupling scheme and thus to allow convergence to a well specified state. Besides the introduction of many body effects this is an important improvement over 
the relativistic single configuration calculations which are restricted to single $\mathrm{jj}$ configurations or to the average energy, i.e. the centre of gravity of all the levels. Neither of these two restrictions is satisfactory for high quality predictions. The method works as follows, the total wavefunction is expressed as :

$$
\Psi=\sum_{i} a_{i}\left|\alpha_{i} J M\right\rangle
$$

where $\alpha_{i}$ stands for all quantum numbers necessary to completely describe the $i$-th configuration of total angular momentum $J$ and projection $M$. Restricting the total Hamiltonian of the system to a sum of oneelectron Dirac operators plus the classical Coulomb repulsion between the electrons, the variational principle is used to determine both the radial functions and the mixing coefficients $a_{i}$ - we will use weights for their square $a_{i}^{2}$ - of the various configurations. After self-consistency, the total energy is corrected by first order perturbation theory for magnetic interaction and retardation in the Coulomb repuision. Both of these contributions have been evaluated exactly for each state considered, with the method we developed recently [15-16], and not within the average configuration approximation.

Furthermore we include an estimation of QED corrections based on hydrogenic results for the selfenergy and on the expectation value of the Uehling potential for the vacuum polarization. Our method of handling these corrections is described elsewhere [16]. In the present use of the MCDF method we include all the electronic configurations arising from the distribution of 3 electrons in the $n \mathrm{~s}, n \mathrm{p}_{1 / 2}, n \mathrm{p}_{3 / 2}$, $(n-1) \mathrm{d}_{3 / 2},(n-1) \mathrm{d}_{5 / 2}$ as well as the nearest $f_{5 / 2}$ and $f_{7 / 2}$ orbitals, where $n$ is the principal quantum number of the outermost occupied orbital. This set of configurations was being chosen to include the most important contributions to correlation effects in the valence shells due to both intra-shell correlation (i.e. mixing between configurations with the same principal quantum number $n$ ) and inter-shell correlation between the valence electrons and the nearest core electrons. We have to point out, nevertheless, that we are restricted to either double excitations or single excitations with a change in the orbital quantum number with the present version of our program, which cannot handle excitations of the type : one electron in an $(n \mathrm{j})$ orbital promoted to an $\left(n^{\prime} \mathrm{j}\right)$ one. We are thus missing some core polarization contributions.

2. Results and discussion. - Calculations, as described in the previous section, have been performed not only for Lawrencium but also for the lighter elements : Sc, Y, La, Lu and Ac, which exhibit analogous valence electrons. For the $J=3 / 2$ even parity state, which in the single configuration approximation will be the $\mathrm{s}^{2} \mathrm{~d}_{3 / 2}{ }^{2} \mathrm{D}_{3 / 2}$ state, the calculation includes 63 (jj) configurations while for the $J=1 / 2$ odd parity state ( $\mathrm{s}^{2} \mathrm{p}_{12}{ }^{2} \mathrm{P}_{1,2}$ state) we have to deal with 35 configurations. This rather large number of configurations is due to the fact that in the relativistic case the only good quantum number is the total angular momentum and not the orbital and spin total angular momenta separately. Being interested in the two $s^{2} p_{1 / 2}$ and $s^{2} d_{3 / 2}$ configurations of $L r$, the only two candidates for the ground state, we carried out the calculations by converging to the states for which these configurations have the largest weight. We found that, except for $\mathrm{Sc}$, this state was the ground state. For $\mathrm{Sc}$ it is known [17] that the lowest $J=1 / 2$ level is almost of pure dsp character and that the lowest odd level has a total angular momentum $J=3 / 2$. These two results are well reproduce by the MCDF method which gives for each of these levels a weight of $99 \%$ for the dsp configuration with the $J=3 / 2$ state $410 \mathrm{~cm}^{-1}$ below the $J=1 / 2$ one (to be compared with the experimental value of $337 \mathrm{~cm}^{-1}$ ). For La, our result is in disagreement with the accepted ground state configuration [18] of the $J=1 / 2$ level. If single configuration calculations, in intermediate coupling, predict that the dsp configuration is lower than the $\mathrm{s}^{2}$ p one, multiconfiguration calculations give a ground state with a predominant $s^{2} p$ character $(66 \%)$ and an important dsp contribution $(24 \%)$. This suggests that further studies of $\mathbf{L a}$ are needed but, as we will see later in the discussion, this will not change our conclusion for $\mathrm{Lr}$.

Before discussing our results more in detail let us first consider the mixing coefficients between the various configurations included in the calculation. For the $J=3 / 2$ even state of all the atoms considered, the $s^{2} d_{32}$ configuration is by far the most important one and contributes between 0.9 to 0.95 to the coefficients $a_{i}$ of equation (1), whereas all other configurations contribute to 0.2 or less each (which means a weight $a_{i}^{2}$ less than $4 \%$ ). The $J=1 / 2$ odd parity state shows a much more complicated behaviour as illustrated by the results given in table I. For La we found very strong contributions of $0.38,0.25$ and 0.17

Table I. - Coefficients $a_{i}$ (according to eq. 1) of the main configurations contributing to the lowest $J=1 / 2^{\circ}$ state for the elements $\mathrm{Sc}, \mathrm{Y}, \mathrm{La}, \mathrm{Lu}, \mathrm{Ac}$ and $\mathrm{Lr}$.

$\begin{array}{ccccccc}\text { jj-configuration } & \mathrm{Sc}\left({ }^{*}\right) & \mathrm{Y} & \mathrm{La} & \mathrm{Lu} & \mathrm{Ac} & \mathrm{Lr} \\ - & - & - & - & - & - & - \\ \mathrm{s}^{2} \mathrm{p}_{1 / 2}^{1} & 0.75 & 0.87 & 0.81 & 0.91 & 0.89 & 0.92 \\ \mathrm{sp}_{1 / 2} \mathrm{~d}_{3 / 2} & 0.12 & 0.30 & 0.38 & 0.26 & 0.30 & 0.28 \\ \mathrm{p}_{1 / 2} \mathrm{p}_{3 / 2}^{2} & 0.18 & 0.17 & 0.14 & 0.15 & 0.13 & 0.13 \\ \mathrm{sp}_{3 / 2} \mathrm{~d}_{3 / 2} & 0.12 & 0.21 & 0.25 & 0.17 & 0.18 & 0.16 \\ \mathrm{p}_{1 / 2} \mathrm{~d}_{3 / 2}^{2} & 0.03 & 0.15 & 0.21 & 0.12 & 0.16 & 0.10 \\ \mathrm{sp}_{3 / 2} \mathrm{~d}_{5 / 2} & 0.14 & 0.17 & 0.17 & 0.15 & 0.15 & 0.11\end{array}$

$\left(^{*}\right)$ Not the ground state but the state for which the configuration $\mathrm{s}^{2} \mathrm{p}$ is the dominant one.

for the $\mathrm{sp}_{1,2} \mathrm{~d}_{3,2}, \mathrm{sp}_{3,2} \mathrm{~d}_{3,2}$ and $\mathrm{sp}_{3,2} \mathrm{~d}_{5,2} \mathrm{jj}$ subconfigurations (which correspond to the single spd non-relativistic $L S$ configuration) besides the main $6 s^{2} 6 p$ configuration. As we have already pointed 
out this result seems to be in contraction with previous ones but it also provides a very good example that the nomenclature usually used to identify the various levels of a complex spectra has, in some cases, to be considered only as a label. Even in Ac the contributions of the three $7 \mathrm{~s} 7 \mathrm{p} 6 \mathrm{djj}$ configurations are still large but the $7 \mathrm{~s}^{2} 7 \mathrm{p}$ configuration has a weight of more than $80 \%$. On the other hand, the increase in the weight of the $s^{2} p$ configuration is only continuous for the elements $\mathrm{Y}, \mathrm{Lu}$ and $\mathrm{Lr}$ on one side and for $\mathrm{La}$ and Ac on the other. This difference of behaviour between these two groups of atoms is also reflected in the expectation $\langle r\rangle$ values of the analogous wavefunctions both for the ground state [2] and the excited ones considered here. The physical reason for this is the SCF influence of the underlying shells. While $4 \mathrm{f}$ and $5 f$ shells are just filled at $\mathrm{Lu}$ and $\mathrm{Lr}, \mathrm{La}$ and $\mathrm{Ac}$ are at the beginning of the f-series. $\mathrm{Y}$ is very comparable with $\mathrm{Lu}$ and $\mathrm{Lr}$ because the $3 \mathrm{~d}$ shell, which has a very similar influence, is just filled. This, of course, is not valid for Sc, as there is no underlying $d$ shell. Consequently, only the elements $\mathrm{Y}$ and $\mathrm{Lu}$ can directly be compared with Lr.

Figure 1 shows the difference in energy, $\Delta_{\mathrm{pd}}$, between the lowest $J=1 / 2^{\circ}$ state and the $J=3 / 2^{\mathrm{e}}$ one. We have also included the best known experimental differences $[18,19]$. In contradiction to all earlier publications [9-12], the experimental values used for comparison, are different in the cases of La and Ac.



Fig. 1. - Energy differences between the lowest $J=1 / 2^{\circ}$ and $J=3 / 2^{\mathrm{e}}$ levels for the elements Sc, Y, La, Lu, Ac and Lr. Crosses are the experimental values $\Delta_{\text {pd }}(\exp )$ and circles are the best calculated values $\Delta_{\mathrm{pd}}$ (th). The experimental value $(-1.5 \pm 1) .10^{3} \mathrm{~cm}^{-1}$ for $\mathrm{Lr}$ is the value predicted in this paper.

For La we have taken the lowest $J=1 / 2^{\circ}$ level, which should correspond to our multiconfiguration ground state, and not the $6 \mathrm{~s}^{2} 6 \mathrm{p}$ state of the Moore's table [18] which was used previously. This is a difference of more than $1100 \mathrm{~cm}^{-1}$.

The estimated value of about $10000 \mathrm{~cm}^{-1}$ for Ac was previously used incorrectly [8]. It is clearly stated in reference [18] that this value is a guess for the ${ }^{2} P_{3 / 2}$ state. We therefore calculated the spin-orbit splitting between the ${ }^{2} \mathbf{P}_{3 / 2}$ and ${ }^{2} \mathbf{P}_{1 / 2}$ states (as the difference between the total energies of two full self-consistent Dirac-Fock calculations) and thus estimate an experimental value of $(6.1 \pm 1.5) \cdot 10^{3} \mathrm{~cm}^{-1}$ for the lowest $J=1 / 2^{\circ}$ level.

Figure 2 shows the double differences $\delta_{\text {pd }}$ between the experimental $\Delta_{\mathrm{pd}}(\exp )$ and calculated $\Delta_{\mathrm{pd}}(\mathrm{th})$

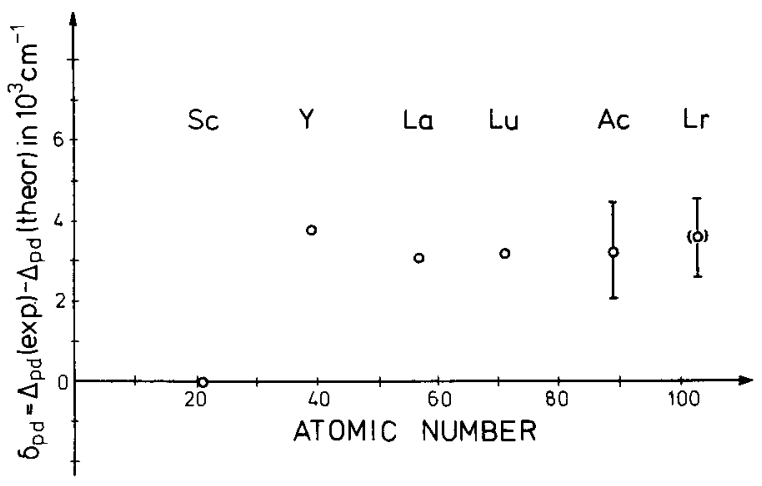

Fig. 2. - Double differences $\delta_{\text {pd }}$ between the experimental $\Delta_{\mathrm{pd}}(\exp )$ and theoretical energy differences $\Delta_{\mathrm{pd}}$ (th) of the lowest $J=1 / 2^{\circ}$ and $J=3 / 2^{\mathrm{e}}$ states for $\mathrm{Sc}, \mathrm{Y}, \mathrm{La}, \mathrm{Lu}, \mathrm{Ac}$. The value shown for $\mathrm{Lr}$ is the extrapolated value.

energies, which are found to be almost constant for the elements $Y$ to Ac. We believe that this relatively large discrepancy between our calculations and the experimental results can mainly be attributed to the influence of core polarization which, as already mentioned, cannot be taken into account by our program. Evidence for this comes both from experimental results and the extended investigation of other possible contributions which we have carried out. The argument on the experimental side [18] is that configurations with two open $\mathrm{s}$ or $\mathrm{d}$ shells are known to be only about $30 \times 10^{3} \mathrm{~cm}^{-1}$ above the ground state of the $J=3 / 2$ level. On the theoretical side we have calculated :

a) The contribution of core excitations of $(n-1) \mathrm{s}$ and $(n-1)$ p electrons. This was done by performing a configuration interaction calculation using the oneelectron wavefunctions we obtained at the end of the self-consistent MCDF calculation. The contribution to $\Delta_{\mathrm{pd}}$ was found to be smaller than $200 \mathrm{~cm}^{-1}$;

b) The exact contribution of the full Breit operator (magnetic and retardation) for the two levels considered. As expected for a neutral heavy atom, the spin-spin and spin-other orbit contributions in the valence shells are small compared to the spin-orbit interaction. The net contribution of the full Breit operator calculated exactly changes $A_{\text {pd }}$ only by a few $\mathrm{cm}^{-1}$ compared to a calculation in which this Breit term was considered in the average configuration approximation.

c) QED corrections for the inner $1 \mathrm{~s}, 2 \mathrm{~s}$ and $2 \mathrm{p}_{1 / 2}$ electrons, where we have verified that these corrections do not contribute to $\Delta_{\mathrm{pd}}$ since the change in the 
valence orbitals is reflected as only a modification of the outer screening for the inner electrons.

Despite of this remaining discrepancy between our calculations and the experimental results, we may conclude on the basis of the almost constant difference we found that the same situation will hold for $\mathrm{Lr}$ in analogy especially with $\mathrm{Y}$ and $\mathrm{Lu}$. Extrapolating the results for the lighter atoms to $\mathrm{Lr}$ we obtain the experimental values shown in figures 1 and 2. This leads to the prediction that the ground state of atomic Lawrencium is the $J=1 / 2^{\circ}$ state $\left({ }^{2} \mathrm{P}_{12}\right)$ with the main contribution from the $7 \mathrm{~s}^{2} 7 \mathrm{p}$ single configuration. The first excited state at about

$$
(1.5 \pm 1) \cdot 10^{3} \mathrm{~cm}^{-1} \text { is the } J=3 / 2^{\mathrm{e}}\left({ }^{2} \mathrm{D}_{3 / 2}\right)
$$

state, which is the ground state for all other analogous elements in the Periodic Table. The reason for this change in the ground state configuration is the increasingly strong influence of relativistic effects on the $\mathrm{p}_{1 / 2}$ electrons with increasing $Z$. For completeness we may add that the spin-orbit splitting between the ${ }^{2} \mathrm{P}_{1: 2}$ and ${ }^{2} \mathrm{P}_{32}$ levels will be in the order of $7900 \mathrm{~cm}^{-1}$ and for the ${ }^{2} \mathrm{D}_{3 / 2}$ and ${ }^{2} \mathrm{D}_{5,2}$ ones of $2400 \mathrm{~cm}^{-1}$. These values have been obtained as the difference of the total energies of the various states.

Thus, atomic Lawrencium is the first element to be known, where relativistic effects directly change the ground state with respect to the normal prediction of the Periodic Table.

Acknowledgments. - We would like to thank Dr. J. B. Mann for many interesting discussions during the early stage of this work. One of us (B.F.) would like to thank DRF-Grenoble for the great hospitality during his stay and the Deutscher Akademischer Austauschdienst for financial support during that time. Parts of the calculations were performed at Gesellschaft für Schwerionenforschung.

\section{References}

[1] FrICKe, B., Progress in Atomic Spectroscopy, Hanle, W. and Kleinpoppen, H., Ed. (Plenum Press New York and London) 1978 , p. 183.

[2] DesclauX, J.-P., Atomic Data and Nuclear Data Tables 12 (1973) 311.

[3] Kim, Y. K. and Desclaux, J.-P., Phys. Rev. Lett. 36 (1976) 139 Aymar, M. and Luc-Koenig, E., Phys. Rev. A 15 (1977) 821.

[4] Johnson, W. R. and Radojevic, V., J. Phys. B 11 (1978) L773.

[5] Fricke, B., Structure and Bonding 2189 (Springer Verlag, Heidelberg) 1975

[6] Madelung, E., Die Mathematischen Hilfsmittel des Physikers, Appendix 15 (Springer Verlag, Berlin) 1936

[7] MaNN, J. B., private communication, 1971

[8] Brewer, L., J. Opt. Soc. 61 (1971) 1101.

[9] Mann, J. B., cited in Seaborg, G. T., Crandall, J. L., Fields, P. R., Ghiorso, A., Keller, O. L. and PenneMAN, R. A., Proceedings IV. Int. Cont. on the Peaceful Use's of Atomic Energy, Geneva, United Nations, A/Conf 49 (1972) 841 (unpublished).
[10] Vander Sluis, K. L. and Nugent, L. J., Phys. Rev. A 6 (1972) 86.

[11] Nugent, L. J., Vander Sluis, K. L., Fricke, B. and Mann, J. B., Phys. Rev. A 9 (1974) 2270.

[12] Mann, J. B., Talk given at the I. Int. Conf. on the Electronic Structure of the Actinides, Argonne, Oct. 1974 (unpublished)

[13] Desclaux, J.-P., Comp. Phys. Comm. 9 (1975) 31.

[14] Froese-Fischer, Ch., The Hartree-Fock Method for Atoms (John Wiley and Sons, New York) 1977.

[15] Cheng, K. T., Desclaux, J.-P. and Kim, Y. K., J. Phys. B 11 (1978) L-359

[16] Desclaux, J.-P., Cheng, K. T. and Kim, Y. K., J. Phys. B 12 (1979) 3819.

[17] Ben Ahmed, Z., Physica 92C (1977) 122.

[18] Moore, C. E., Atomic Energy Levels, Vol. I, II and III, Natl. Bur. Std. U.S. Circ. 467, NSRDS-NBS35, Edition 1970.

[19] Pinnington, E. H., Can. J. Phys. 41 (1963) 1294. 